\title{
Out-of-Time-Ordered Correlator in Many-Body Localized Systems
}

\author{
Yichen Huang (黄溢辰) ${ }^{*}$ Yong-Liang Zhang (张涌良) † and Xie Chen (陈谐) \\ Department of Physics and Institute for Quantum Information and Matter, \\ California Institute of Technology, Pasadena, California 91125, USA
}

(Dated: August 10, 2016)

\begin{abstract}
In many-body localized systems, propagation of information forms a light cone that grows logarithmically with time. However, local changes in energy or other conserved quantities are expected to spread only within a finite distance. Is it possible to detect the logarithmic light cone using local observables, starting from thermal states or eigenstates? Here we show that this is indeed the case, but only in an out-of-time-ordered way. In particular, we numerically calculate various correlators in a prototypical model of many-body localization. While correlators of local operators $A(t=0)$ and $B(t>0)$ ordered in time sequence - e.g., $A(t=0) B(t>0)$ - are not sensitive to the unbounded information propagation, those with out-of-time order - e.g., $A(t=0) B(t>0) A(t=0) B(t>0)-$ can detect the logarithmic light cone. We demonstrate the connection between out-of-time-ordered correlators and the Lieb-Robinson bound in many-body localized systems, and explain how to saturate the latter by measuring the involved commutator on specially designed initial states. Moreover, out-of-time-ordered correlators also allow us to study the temperature dependence of the light cones and we observe an increase of propagation velocity with increasing temperature.
\end{abstract}

In the presence of disorder, localization can occur not only in single-particle quantum systems [1, but also in interacting many-body systems 2 10. The former is known as Anderson localization, and the latter is called many-body localization (MBL). Neither Anderson localized nor MBL systems conduct heat, charge, or other local conserved quantities. Indeed, changes in energy or charge at position $\vec{x}=0$ from equilibrium can propagate and lead to changes in the corresponding quantity only within a finite distance with $|\vec{x}|<L_{0}$, where $L_{0}$ is called the localization length. Note that entanglement also remains bounded after a local perturbation from eigenstates [11]. Other transport-related measurements only demonstrate strictly local effects: e.g., fluctuation of conserved quantities remains finite after a global quench [12 and domain wall configuration does not completely melt after a sufficiently long time 13 .

The entanglement spreading, i.e., the propagation of information, on the other hand, is in stark contrast to the transport of conserved quantities. Although information propagation is strictly local in Anderson localized systems [14, 15, entanglement in MBL systems grows without bound after a global quench [12, 16, 23. In particular, it was shown that if the initial state is prepared in a random product state by a random field and interaction is turned on at $t=0$, entanglement of half of the chain grows logarithmically with time. This result suggests that in MBL systems, information propagation is not restricted to a finite region, as otherwise entanglement would saturate at a finite value. Instead, information propagates throughout the system, although at a very slow rate [24, 25]. This feature distinguishes MBL from Anderson localization.

The propagation of information can be formalized by adapting the Lieb-Robinson bound [26, 28, to the context of MBL systems [29]. In particular, one might expect a logarithmic light cone in the sense that the operator norm of the commutator of two local (w.l.o.g.) Hermitian operators $A$ and $B$ with time separation $t$ and spatial separation $x$ decays exponentially outside a light cone whose radius is given by $|x| \sim \log |t|$. That is,

$$
\|[A(0,0), B(x, t)]\| \leq c e^{-a(|x|-b \log |t|)}
$$

for some positive constants $a, b, c$. Here, $B(x, t)$ is the time evolved operator in the Heisenberg picture $B(x, t)=$ $e^{i H t} B(x, 0) e^{-i H t}$, and $\|\cdot\|$ denotes the operator norm (the maximum singular value) of an operator.

Can the unbounded information propagation, or more specifically, the logarithmically growing light cone be detected with local observables instead of entanglement? One straightforward idea is to directly measure the Hermitian commutator $i[A(0,0), B(x, t)]$ in the LiebRobinson bound (Eq. 1) on thermal states or eigenstates. This method does not work, because it is related to the linear response measured by $B$ after the local unitary perturbation $V=e^{-i \tau A}$ for small $\tau$ via the Kubo formula,

$$
\left\langle V^{\dagger} B(x, t) V\right\rangle-\langle B(x, t)\rangle=\tau\langle i[A, B(x, t)]\rangle+O\left(\tau^{2}\right) .
$$

Note that $V$ and $B$ can be, although do not have to be, chosen as the operations of changing and measuring local conserved quantities, respectively. Hence outside the localization length $L_{0}$, there is approximately no change after perturbation for the evolved thermal state $e^{-i H t} V\left(e^{-\beta H} / \operatorname{tr}\left(e^{-\beta H}\right)\right) V^{\dagger} e^{i H t}$ or eigenstate $e^{-i H t} V\left|\psi_{k}\right\rangle\left(\left|\psi_{k}\right\rangle\right.$ is an eigenstate of the Hamiltonian).

The crucial feature in the Lieb-Robinson bound is the degree of non-commutativity between operators $A(0,0)$ and $B(x, t)$. In order to detect the logarithmic light cone, we consider the out-of-time-ordered (OTO) correlator 30 33] of the form $A(0,0) B(x, t) A(0,0) B(x, t)$ for generic local observables. It plays a crucial role in investigating the butterfly effect and scrambling in quantum 
chaotic systems especially the black holes 30 38. We observe that OTO correlators can detect the logarithmic light cone. To understand this, we discuss its relation to the square of the commutator $-[A(0,0), B(x, t)]^{2}$ and furthermore to the Frobenius norm of $[A(0,0), B(x, t)]$ which is expected to behave similarly to the operator norm in the Lieb-Robinson bound in Eq. 1. In addition, OTO correlators allow us to observe the temperature dependence of the logarithmic light cones.

The fact that the Lieb-Robinson bound (Eq. 1) predicts a logarithmic light cone while the expectation value of $[A(0,0), B(x, t)]$ on thermal states or eigenstates approaches zero outside a finite distance raises the question of whether the Lieb-Robinson bound (Eq. 11 is saturated when measured on some set of initial states. Of course, it is saturated when measured on the eigenstate of $[A(0,0), B(x, t)]$ with the largest eigenvalue in magnitude, but such states are not very physical. Is it possible to saturate the Lieb-Robinson bound (Eq. 1) on physically motivated states? We provide numerical and analytical evidences that this is not the case on the eigenstates of the Hamiltonian, any mixture of them, or random product states.

Finally, we compare the above observations on MBL systems with Anderson localized systems. In Anderson localized systems, all response functions including information propagation are localized. That is, the system has a strictly non-expanding light cone and OTO correlators do not decay outside a finite distance, which we show explicitly using a random-field $X Y$ model.

Out-of-time-ordered correlator as a probe.-How do we measure the logarithmic light cone in an MBL system using local observables starting from thermal states or eigenstates? To answer this question, we consider the spin-1/2 random-field Heisenberg chain and simulate its dynamics using exact diagonalization. The Hamiltonian is

$$
H=\sum_{j=1}^{L-1}\left(\sigma_{j}^{x} \sigma_{j+1}^{x}+\sigma_{j}^{y} \sigma_{j+1}^{y}+\sigma_{j}^{z} \sigma_{j+1}^{z}\right)+\sum_{j=1}^{L} h_{j} \sigma_{j}^{z},
$$

where $\sigma_{j}^{x}, \sigma_{j}^{y}, \sigma_{j}^{z}$ are the Pauli matrices at the site $j$, and $h_{j}$ 's are independent and identically distributed (i.i.d.) uniform random variables on the interval $[-h, h]$. This model is known to be in the MBL phase for $h \gtrsim 7$ [39]41, and we fix $h=16$. Most data presented below are for $L=$ 10 , but we keep doing finite-size scaling to guarantee that the system size we use is large enough for our purposes. For the ease of presentation, data for finite-size scaling are not shown unless necessary.

Suppose the system is prepared in the thermal state or a random eigenstate. Their expectations are given by

$$
\begin{aligned}
\langle\hat{O}\rangle_{\text {th }} & =\operatorname{tr}\left(e^{-\beta H} \hat{O}\right) / \operatorname{tr}\left(e^{-\beta H}\right), \\
\langle\hat{O}\rangle_{\text {eig }} & =\left\langle\psi_{k}|\hat{O}| \psi_{k}\right\rangle,
\end{aligned}
$$
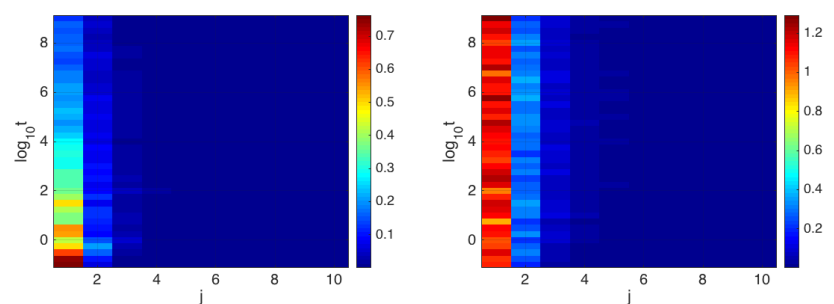

FIG. 1. (Color online) Color plots of $\left|\left\langle i\left[\sigma_{1}^{x}, \sigma_{j}^{x}(t)\right]\right\rangle_{\mathrm{th}}\right|$ for $\beta=0.1$ (left) and $\left|\left\langle i\left[\sigma_{1}^{x}, \sigma_{j}^{x}(t)\right]\right\rangle_{\text {eig }}\right|$ (right), averaged over 100 samples. The distance remains finite for large $t$.

where $\beta=1 / T$ is the inverse temperature, and $\left|\psi_{k}\right\rangle$ is a random eigenstate of $H$.

We calculate the response of the operator $B_{j}=\sigma_{j}^{x}$ for $j=1,2, \ldots, L$ to perturbation at the left end of the chain $A=\sigma_{1}^{x}$. The result is plotted as a function of $j$ and $t$ in Fig. 1. From this figure, it is straightforward to see that changes in $B$ in response to $A$, whether in a thermal state or in a random eigenstate, is restricted within a finite distance. We observe similar behavior for other choices of local operators $A$ and $B$. As we mentioned before, this phenomenon can be explained by the Kubo formula (2) and localization.

We now consider the OTO operator $\sigma_{1}^{x} \sigma_{j}^{x}(t) \sigma_{1}^{x} \sigma_{j}^{x}(t)$, which is very close to $I$ if $\left[\sigma_{1}^{x}, \sigma_{j}^{x}(t)\right] \approx 0$, i.e., if the site $j$ at time $t$ is outside the light cone originating at the first site. We can see from Fig. 2 (upper panels) that this OTO correlator clearly visualizes an unbounded light cone. Specifically, the radius of the light cone grows as $b \log t$ for some positive constant $b$ when $t$ is large. We also calculate the OTO operator $A B_{j}(t) A B_{j}(t)$ for $A=\sigma_{1}^{z}, B_{j}=\sigma_{j}^{z}$ (lower left panel) and $A=\left(\sigma_{1}^{x}+\sigma_{1}^{z}\right) / \sqrt{2}, B_{j}=\left(\sigma_{j}^{x}+\sigma_{j}^{z}\right) / \sqrt{2}$ (lower right panel). The light cone is visible in the lower right panel $\left(A=\left(\sigma_{1}^{x}+\sigma_{1}^{z}\right) / \sqrt{2}, B_{j}=\left(\sigma_{j}^{x}+\sigma_{j}^{z}\right) / \sqrt{2}\right)$ but is not very clear in the lower left panel $\left(A=\sigma_{1}^{z}, B_{j}=\sigma_{j}^{z}\right)$. This is because $\sigma_{j}^{z}$ has a large overlap with the local integrals of motion [22, 42] and therefore does not 'scramble' 32, 33, 36] very strongly. Furthermore, the OTO correlator $\operatorname{Re}\left\langle A B_{j}(t) A B_{j}(t)\right\rangle_{\text {eig }}$ decays (does not decay) to 0 for very large $t$ in the upper (lower) right panel, where Re denotes the real part. This is because $\sigma_{j}^{x}\left(\sigma_{j}^{x}+\sigma_{j}^{z}\right)$ has a vanishing (finite) overlap with the local integrals of motion. We see that the OTO correlator $\operatorname{Re}\left\langle\sigma_{1}^{z} \sigma_{j}^{z}(t) \sigma_{1}^{z} \sigma_{j}^{z}(t)\right\rangle_{\text {eig }}$ almost does not decay.

Why does OTO correlation behave differently from normal response functions? Loosely speaking, normal response functions measures the spreading of physical quantities like energy or charge, while OTO correlation measures the spreading of information. In particular, we show below that the OTO correlator is connected to the Lieb-Robinson bound (Eq. 1) describing a light cone that grows logarithmically in time in an MBL phase.

The OTO correlator can be obtained by expanding the 

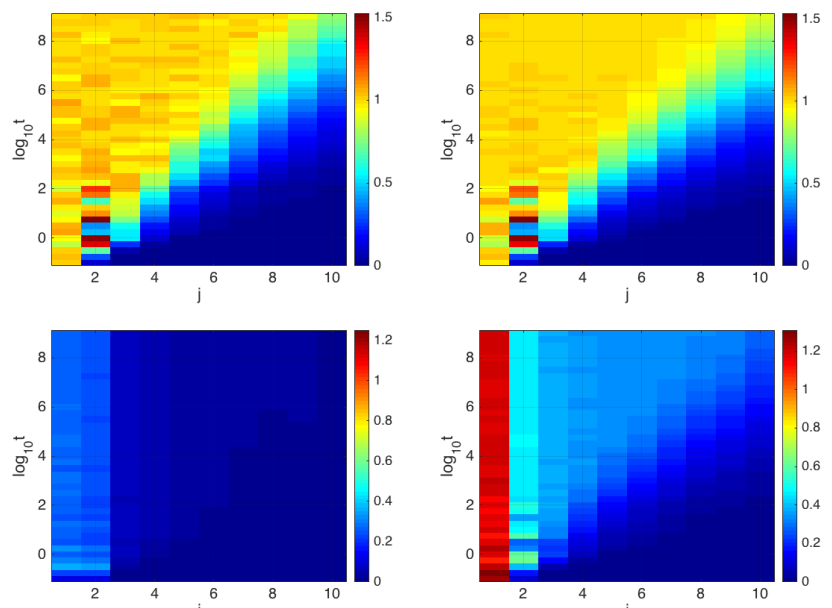

FIG. 2. (Color online) Color plots of $1-\operatorname{Re}\left\langle\sigma_{1}^{x} \sigma_{j}^{x}(t) \sigma_{1}^{x} \sigma_{j}^{x}(t)\right\rangle_{\mathrm{th}}$ for $\beta=0.1$ (upper left panel), $1-\operatorname{Re}\left\langle\sigma_{1}^{x} \sigma_{j}^{x}(t) \sigma_{1}^{x} \sigma_{j}^{x}(t)\right\rangle_{\text {eig }}$ (upper right panel), $1-\operatorname{Re}\left\langle\sigma_{1}^{z} \sigma_{j}^{z}(t) \sigma_{1}^{z} \sigma_{j}^{z}(t)\right\rangle_{\text {eig }}$ (lower left panel $)$, and $1-\operatorname{Re}\left\langle\left(\sigma_{1}^{x}+\sigma_{1}^{z}\right)\left(\sigma_{j}^{x}(t)+\sigma_{j}^{z}(t)\right)\left(\sigma_{1}^{x}+\sigma_{1}^{z}\right)\left(\sigma_{j}^{x}(t)+\right.\right.$ $\left.\left.\sigma_{j}^{z}(t)\right)\right\rangle_{\text {eig }} / 4$ (lower right panel), averaged over 100 samples. The distance in all but the lower left panels grows as $b \log t$ when $t$ is large. At a numerical level, it is not clear whether $1-\operatorname{Re}\left\langle\sigma_{1}^{z} \sigma_{j}^{z}(t) \sigma_{1}^{z} \sigma_{j}^{z}(t)\right\rangle_{\text {eig }}$ can be used to detect the logarithmic light cone.

square of the commutator

$$
-\left[\sigma_{1}^{x}, \sigma_{j}^{x}(t)\right]^{2}=2-\sigma_{1}^{x} \sigma_{j}^{x}(t) \sigma_{1}^{x} \sigma_{j}^{x}(t)-\sigma_{j}^{x}(t) \sigma_{1}^{x} \sigma_{j}^{x}(t) \sigma_{1}^{x}
$$

Indeed, the thermal average $\left\langle-\left[\sigma_{1}^{x}, \sigma_{j}^{x}(t)\right]^{2}\right\rangle_{\text {th }}$ at infinite temperature $\beta=0$ is the squared Frobenius norm of the commutator $\left[\sigma_{1}^{x}, \sigma_{j}^{x}(t)\right]$ divided by $2^{L}$. This is closely related to the Lieb-Robinson bound (Eq. 1) which states that the operator norm of $\left[\sigma_{1}^{x}, \sigma_{j}^{x}(t)\right]$, i.e., the largest (in magnitude) eigenvalue of $\left[\sigma_{1}^{x}, \sigma_{j}^{x}(t)\right]$, decays exponentially outside a light cone that grows logarithmically with time. Plotting the operator norm of $\left[\sigma_{1}^{x}, \sigma_{j}^{x}(t)\right]$ in Fig. 3 (right panel), we clearly observe that the bound (Eq. 1) can indeed be saturated. Therefore, while the definitions of the Frobenius and operator norms are different, they behave very similarly in the present context. In this way, we have established a connection between the logarithmic spreading of OTO correlation and the logarithmic light cone described in the Lieb-Robinson bound (Eq. 1) in MBL systems.

Note the sharp contrast between the expectation of $-\left[\sigma_{1}^{x}, \sigma_{j}^{x}(t)\right]^{2}$ as shown in Fig. 2 and that of $\left[\sigma_{1}^{x}, \sigma_{j}^{x}(t)\right]$ as shown in Fig. 11. The fact that the former demonstrates unbounded spreading while the latter does not is related to the strong sign oscillation in the eigenvalues of $\left[\sigma_{1}^{x}, \sigma_{j}^{x}(t)\right]$, which upon taking the expectation (either with respect to a thermal state or a random eigenstate) cancel among themselves. The eigenvalues of $-\left[\sigma_{1}^{x}, \sigma_{j}^{x}(t)\right]^{2}$ on the other hand, are always positive and contribute additively when taking the expectation
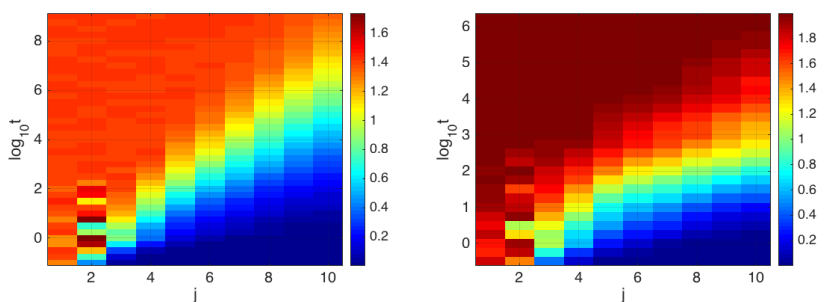

FIG. 3. (Color online) Color plots of the Frobenius norm divided by $2^{L / 2}$ (left) and operator (right) norm of $\left[\sigma_{1}^{x}, \sigma_{j}^{x}(t)\right]$, averaged over 100 samples. Both quantities spread to a distance which grows logarithmically in time.

values.

OTO correlators also allow us to observe the temperature dependence of logarithmic light cones (this problem for linear light cones in ergodic systems has been studied; see, e.g., 38]). In particular, we study how the light cone boundary $x \sim b \log _{10} t$ depends on temperature. To this end, we choose a threshold value $\epsilon$ and extract $b$ by solving the relation between $j$ and $t$ from the equation

$$
1-\operatorname{Re}\left\langle\sigma_{1}^{x} \sigma_{j}^{x}(t) \sigma_{1}^{x} \sigma_{j}^{x}(t)\right\rangle_{\mathrm{th}}=\epsilon .
$$

It should be noted that $b$ depends on both $\epsilon$ and $\beta$. For fixed $\epsilon$, we observe that $b$ decreases as $\beta$ increases. This observation is similar to that found in 38. for systems with a holographic dual. On the other hand, this behavior can also be interpreted in terms of the localization dynamics of the model. It is know that for smaller $h$ there are mobility edges to thermal phases in the middle of the spectrum 40. For our choice of $h$, the system does not have mobility edges but one might still expect the system to be more localized when temperature goes to either positive zero (ground state) or negative zero (highest excited state) from infinity. Therefore, when $\beta$ increases from 0 to finite positive value, $b$ is expected to decrease as shown in Fig. 4.

Similarly, OTO correlators can detect mobility edges. In particular, when we tune energy density (or temperature) across a mobility edge from localized to delocalized parts of the spectrum, we expect that the light cone (as visualized by OTO correlators) changes from logarithmic to power law.

Saturation of Lieb-Robinson bound. - The fact that the operator norm of $\left[\sigma_{1}^{x}, \sigma_{j}^{x}(t)\right]$ saturates the logarithmic light cone, as shown in Figure 3 (right panel), is equivalent to the fact that the expectation value of $\left[\sigma_{1}^{x}, \sigma_{j}^{x}(t)\right]$ taken with respect to its eigenstate corresponding to the largest (in magnitude) eigenvalue saturates the logarithmic light cone. In Fig. 5 (upper left panel), we plot the expectation value of $\left[\sigma_{1}^{x}, \sigma_{j}^{x}(t)\right]$ taken on the largest (in magnitude) eigenvalue eigenstate of the commutator on the logarithmic light cone. In particular, we roughly locate the boundary of the logarithmic light cone in Fig. 3 (right panel) as $t=10^{0.4 j+0.5}$. We choose $\left|\psi_{j}\right\rangle$ to be 


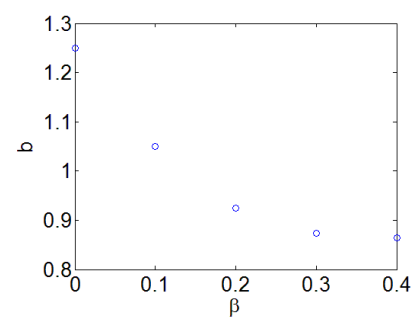

FIG. 4. (Color online) The light cone $x \sim b \log _{10} t$ at various inverse temperature $\beta$. The data in this plot are obtained by solving (7) for $\epsilon=1 / 2$. Note that the color plots for $\beta=0,0.1$ are shown in Fig. 2 (upper panels), and the color plots for other $\beta$ are not shown. It should be clear that $b$ decreases as $\beta$ increases for fixed $\epsilon$.

the eigenstate of $\left[\sigma_{1}^{x}, \sigma_{j}^{x}\left(t=10^{0.4 j+0.5}\right)\right]$ with the largest (in magnitude) eigenvalue. We do see a logarithmic light cone. This is in contrast to the observation in Fig. 1 that the expectation of $\left[\sigma_{1}^{x}, \sigma_{j}^{x}(t)\right]$ with respect to the thermal states or eigenstates of the Hamiltonian cannot visualize the logarithmic light cone.

Since the eigenstates of the commutator are arguably not physically motivated, we would like to investigate whether the logarithmic light cone can be detected through expectation value of $i\left[\sigma_{1}^{x}, \sigma_{j}^{x}(t)\right]$ (which describes linear response via the Kubo formula (Eq. 2p) on random product states, which constitute an important class of physically accessible states both experimentally and numerically. The result is plotted in the lower panels of Fig. 5 Although there might seem to be an unbounded light cone in each of these panels, we argue that our numerical results indeed point to a finite light cone in the thermodynamic limit by doing finite-size scaling. (On the other hand, as mentioned above, we have done finitesize scaling for all previous plots and do not encounter similar problems.) To this end, we consider the quantity $\max _{t}\left|\left\langle\left[\sigma_{1}^{x}, \sigma_{j}^{x}(t)\right]\right\rangle\right|$, which measures the maximum "signal" that can ever be detected by $\sigma_{j}^{x}$ at any time (see Eq. 2). We emphasize that this quantity does not describe whether the light cone is logarithmic, but only detects whether the light cone is bounded. Specifically, if it becomes vanishing and even decays exponentially at large distance, then the light cone should be considered finite. Fig. 5 (upper right panel) provides evidences that in the thermodynamic limit the maximum of the expectation of $i\left[\sigma_{1}^{x}, \sigma_{j}^{x}(t)\right]$ with respect to random product states decays exponentially to 0 as $j$ increases. In this sense, the light cone as seen from random product states is indeed finite. In the appendix, we calculate analytically the linear response of some product states using the phenomenological "l-bits" description of MBL systems [22, which qualitatively explains the behavior shown in Fig. 5.

Comparison with Anderson localization.-Information propagation forms a finite light cone in a single-particle
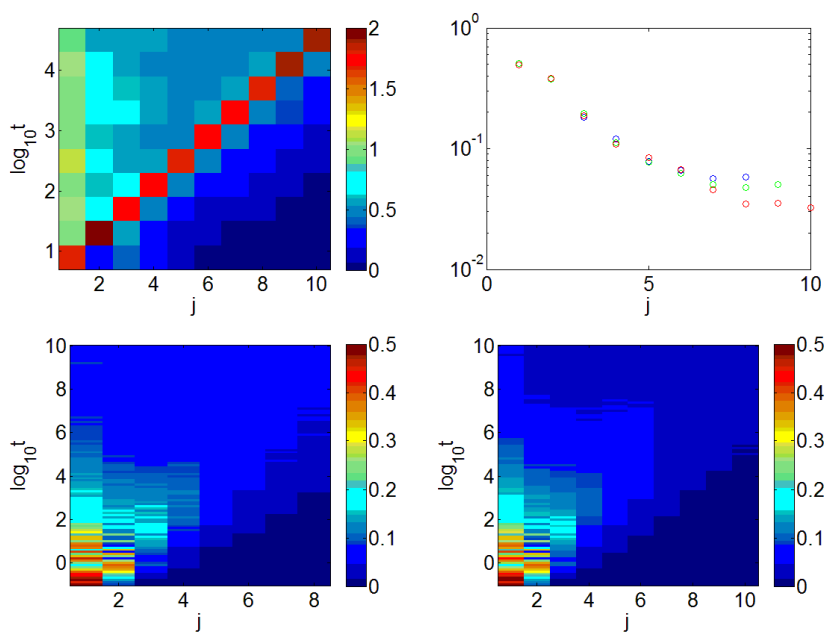

FIG. 5. (Color online) (upper left) Color plot of $\left|\left\langle\psi_{j}\left|\left[\sigma_{1}^{x}, \sigma_{j}^{x}(t)\right]\right| \psi_{j}\right\rangle\right|$, averaged over 100 samples. We choose $\left|\psi_{j}\right\rangle$ to be the eigenstate of $\left[\sigma_{1}^{x}, \sigma_{j}^{x}\left(t=10^{0.4 j+0.5}\right)\right]$ with the largest (in magnitude) eigenvalue. (upper right) Finite size scaling of $\max _{t}\left|\left\langle\left[\sigma_{1}^{x}, \sigma_{j}^{x}(t)\right]\right\rangle\right|$ with respect to random product states for $L=8$ (blue), 9 (green), 10 (red), average over 500 samples. It appears that the quantity under consideration decays exponentially as $j$ (distance) increases. (lower panels) Color plots of $\left|\left\langle\left[\sigma_{1}^{x}, \sigma_{j}^{x}(t)\right]\right\rangle\right|$ with respect to random product states for $L=8$ and $L=10$.

Anderson insulator. This intuition can be formalized by a strictly local Lieb-Robinson bound [14]

$$
\|[A(0,0), B(x, t)]\| \leq c e^{-a|x|},
$$

and by the fact that entanglement remains bounded if the initial state is a product state [15].

All previously studied quantities should visualize a finite light cone in the long time limit. Thus, OTO correlators provide a way to distinguish MBL from Anderson localization. To see this explicitly, we consider the random-field XX chain as a model of Anderson localization:

$$
H=\sum_{j=1}^{L-1}\left(\sigma_{j}^{x} \sigma_{j+1}^{x}+\sigma_{j}^{y} \sigma_{j+1}^{y}\right)+\sum_{j=1}^{L} h_{j} \sigma_{j}^{z},
$$

where $h_{j}$ 's are i.i.d. uniform random variables on the interval $[-h, h]$. We still take $h=16$. This model can be mapped to a free-fermion hopping model in a random potential. We calculate the same quantities as before and plot them in Fig. 6, which clearly shows that both conserved quantity propagation and information propagation are limited to a finite region.

Therefore, the intuition that localization means no information propagation holds in Anderson localized phases, but not in MBL phases.

Conclusion. - To summarize, although an MBL phase is characterized by a lack of transport, information still propagates throughout the system after a local perturbation which can be detected using OTO correlators on 

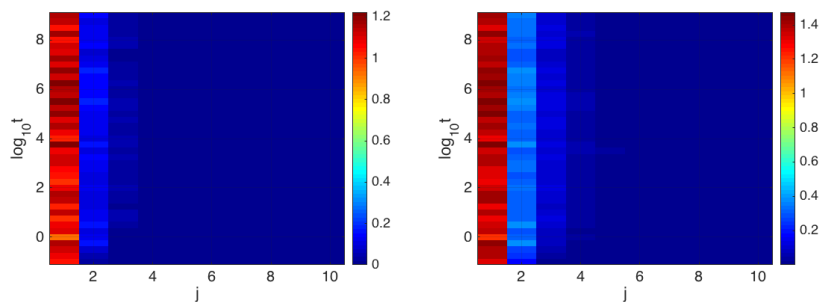

FIG. 6. (Color online) Color plots of $1-$ $\operatorname{Re}\left\langle\sigma_{1}^{x} \sigma_{j}^{x}(t) \sigma_{1}^{x} \sigma_{j}^{x}(t)\right\rangle_{\text {eig }}$ (left) and the operator norm of $\left[\sigma_{1}^{x}, \sigma_{j}^{x}(t)\right]$ (right) for the Anderson localized model (9p) averaged from 100 samples. The light cone remains finite in the long time limit.

eigenstates or thermal states, as we have shown above. Furthermore, we find that on specially designed initial states, even linear response functions can have nonlocalized behavior, although those on random product state still decays exponentially with distance. Whether linear response function on other physically motivated states can exceed strict localization and even achieve logarithmic light cone is an interesting question for future study.

Moreover, we study the temperature dependence of the logarithmic light cones using OTO correlators and observe an increase in propagation velocity with temperature. While this observation is similar to that in [38, the possible interpretation we discussed above in terms of localization dynamics suggests that this behavior may change depending on the mobility structure of the system. We leave this possibility for future study.

OTO correlators have been extensively studied in the context of quantum gravity [30 33] where it is used to study the chaotic dynamics and scrambling behavior of black holes through the AdS-CFT duality. Here we present an example where OTO correlators provide a useful probe of the dynamics of normal quantum mechanical system in general, whether they have a holographic dual or not.

The usefulness of OTO correlators is not restricted to localized systems but applies to ergodic systems as well. In particular, in non-integrable diffusive systems, we expect that OTO correlators can be used to access the linear light cone described in the Lieb-Robinson bound while normal time ordered correlation functions is only sensitive to the diffusive transport at a much lower speed. Furthermore, similar to MBL cases, linear light cone in non-integrable diffusive systems may be detected from linear response of e.g. energy, provided that the initial states are specially designed. An extensive study of ergodic systems is going to be presented elsewhere.

Note added. - Shortly before and after we posted the original version of this paper on arXiv, we became aware of two related works, one by Fan et al. [43] and one by Swingle and Chowdhury. They also studied OTO corre- lators in MBL systems.

We would like to thank Yoni BenTov, Manuel Endres, Alexei Y. Kitaev, Joel E. Moore, and Rahul Nandkishore for inspiring discussions. In particular, we would like to acknowledge discussions with Yingfei $\mathrm{Gu}$, which led to the study of the temperature dependence of logarithmic light cones. Y.H. and X.C. acknowledge funding provided by the Institute for Quantum Information and Matter, an NSF Physics Frontiers Center (NSF Grant PHY1125565) with support of the Gordon and Betty Moore Foundation (GBMF-2644). X.C. is also supported by the Walter Burke Institute for Theoretical Physics.

$\begin{array}{ll}* & \text { ychuang@caltech.edu } \\ \dagger & \text { ylzhang91@caltech.edu } \\ & \text { xiechen@caltech.edu }\end{array}$

[1] P. W. Anderson, "Absence of diffusion in certain random lattices," Phys. Rev. 109, 1492-1505 (1958).

[2] D. M. Basko, I. L. Aleiner, and B. L. Altshuler, "Metalinsulator transition in a weakly interacting many-electron system with localized single-particle states," Ann. Phys. 321, 1126-1205 (2006).

[3] J. Z. Imbrie, "On many-body localization for quantum spin chains," J. Stat. Phys. 163, 998-1048 (2016).

[4] J. Z. Imbrie, "Diagonalization and many-body localization for a disordered quantum spin chain," Phys. Rev. Lett. 117, 027201 (2016)

[5] M. Schreiber, S. S. Hodgman, P. Bordia, H. P. Lüschen, M. H. Fischer, R. Vosk, E. Altman, U. Schneider, and I. Bloch, "Observation of many-body localization of interacting fermions in a quasirandom optical lattice," Science 349, 842-845 (2015).

[6] J.-y. Choi, S. Hild, J. Zeiher, P. Schauß, A. RubioAbadal, T. Yefsah, V. Khemani, D. A. Huse, I. Bloch, and C. Gross, "Exploring the many-body localization transition in two dimensions," Science 352, 1547-1552 (2016).

[7] J. Smith, A. Lee, P. Richerme, B. Neyenhuis, P. W. Hess, P. Hauke, M. Heyl, D. A. Huse, and C. Monroe, "Many-body localization in a quantum simulator with programmable random disorder," Nat. Phys. 12 (2016).

[8] R. Nandkishore and D. A. Huse, "Many-body localization and thermalization in quantum statistical mechanics," Annu. Rev. Condens. Matter Phys. 6, 15-38 (2015).

[9] E. Altman and R. Vosk, "Universal dynamics and renormalization in many-body-localized systems," Annu. Rev. Condens. Matter Phys. 6, 383-409 (2015).

[10] Romain Vasseur and Joel E Moore, "Nonequilibrium quantum dynamics and transport: from integrability to many-body localization," J. Stat. Mech. 2016, 064010 (2016).

[11] J. A. Kjäll, J. H. Bardarson, and F. Pollmann, "Manybody localization in a disordered quantum Ising chain," Phys. Rev. Lett. 113, 107204 (2014)

[12] J. H. Bardarson, F. Pollmann, and J. E. Moore, "Unbounded growth of entanglement in models of many-body localization," Phys. Rev. Lett. 109, 017202 (2012).

[13] J. Hauschild, F. Heidrich-Meisner, and F. Pollmann, "Domain-wall melting as a probe of many-body local- 
ization," arXiv: 1605.05574 (2016), arXiv:1605.05574.

[14] E. Hamza, R. Sims, and G. Stolz, "Dynamical localization in disordered quantum spin systems," Commun. Math. Phys. 315, 215-239 (2012).

[15] H. Abdul-Rahman, B. Nachtergaele, R. Sims, and G. Stolz, "Entanglement dynamics of disordered quantum XY chains," Lett. Math. Phys. 106, 649-674 (2016).

[16] G. De Chiara, S. Montangero, P. Calabrese, and R. Fazio, "Entanglement entropy dynamics of Heisenberg chains," J. Stat. Mech. 2006, P03001 (2006).

[17] M. Znidaric, T. Prosen, and P. Prelovsek, "Many-body localization in the Heisenberg $X X Z$ magnet in a random field," Phys. Rev. B 77, 064426 (2008)

[18] A. Nanduri, H. Kim, and D. A. Huse, "Entanglement spreading in a many-body localized system," Phys. Rev. B 90, 064201 (2014)

[19] Ronen Vosk and Ehud Altman, "Many-body localization in one dimension as a dynamical renormalization group fixed point," Phys. Rev. Lett. 110, 067204 (2013).

[20] Ronen Vosk and Ehud Altman, "Dynamical quantum phase transitions in random spin chains," Phys. Rev. Lett. 112, 217204 (2014).

[21] Maksym Serbyn, Z. Papić, and Dmitry A. Abanin, "Universal slow growth of entanglement in interacting strongly disordered systems," Phys. Rev. Lett. 110, 260601 (2013)

[22] D. A. Huse, R. Nandkishore, and V. Oganesyan, "Phenomenology of fully many-body-localized systems," Phys. Rev. B 90, 174202 (2014)

[23] Brian Swingle, "A simple model of many-body localization," arXiv: 1307.0507 (2013).

[24] M Friesdorf, A H Werner, M Goihl, J Eisert, and W Brown, "Local constants of motion imply information propagation," New J. Phys. 17, 113054 (2015)

[25] M. Goihl, M. Friesdorf, A. H. Werner, W. Brown, and J. Eisert, "Experimentally accessible witnesses of manybody localisation," ArXiv:1601.02666.

[26] E. H. Lieb and D. W. Robinson, "The finite group velocity of quantum spin systems," Commun. Math. Phys. 28, 251-257 (1972).

[27] B. Nachtergaele and R. Sims, "Lieb-robinson bounds and the exponential clustering theorem," Commun. Math. Phys. 265, 119-130 (2006)

[28] M. B. Hastings and T. Koma, "Spectral gap and exponential decay of correlations," Commun. Math. Phys. 265, 781-804 (2006)

[29] I. H. Kim, A. Chandran, and D. A. Abanin, "Local integrals of motion and the logarithmic lightcone in manybody localized systems," arXiv: 1412.3073 (2014).

[30] Daniel A. Roberts, Douglas Stanford, and Leonard Susskind, "Localized shocks," arXiv: 1409.8180 (2014).

[31] Daniel A. Roberts and Douglas Stanford, "Twodimensional conformal field theory and the butterfly effect," arXiv: 1412.5123 (2014).

[32] Juan Maldacena, Stephen H. Shenker, and Douglas Stanford, "A bound on chaos," arXiv: 1503.01409 (2015)

[33] Pavan Hosur, Xiao-Liang Qi, Daniel A. Roberts, and Beni Yoshida, "Chaos in quantum channels," arXiv: 1511.04021 (2015).

[34] Stephen H. Shenker and Douglas Stanford, "Black holes and the butterfly effect," arXiv: 1306.0622 (2013).

[35] Stephen H. Shenker and Douglas Stanford, "Multiple shocks," arXiv: 1312.3296 (2013).
[36] Stephen H. Shenker and Douglas Stanford, "Stringy effects in scrambling," arXiv: 1412.6087 (2014).

[37] Brian Swingle, Gregory Bentsen, Monika Schleier-Smith, and Patrick Hayden, "Measuring the scrambling of quantum information," arXiv: 1602.06271 (2016)

[38] Daniel A. Roberts and Brian Swingle, "Lieb-robinson and the butterfly effect," arXiv: 1603.09298 (2016).

[39] Arijeet Pal and David A. Huse, "Many-body localization phase transition," Phys. Rev. B 82, 174411 (2010).

[40] D. J. Luitz, N. Laflorencie, and F. Alet, "Many-body localization edge in the random-field Heisenberg chain," Phys. Rev. B 91, 081103 (2015).

[41] M. Serbyn, Z. Papić, and D. A. Abanin, "Criterion for many-body localization-delocalization phase transition," Phys. Rev. X 5, 041047 (2015)

[42] M. Serbyn, Z. Papić, and D. A. Abanin, "Local conservation laws and the structure of the many-body localized states," Phys. Rev. Lett. 111, 127201 (2013).

[43] R. Fan, P. Zhang, H. Shen, and H. Zhai, "Outof-time-order correlation for many-body localization," ArXiv:1608.01914.

\section{LINEAR RESPONSE FOR NON-EIGENSTATES}

In this section, we compute analytically the linear response of a particular class of non-eigenstates using the phenomenological description of the MBL system in terms of the "l-bits" 22. The analytical result supports our numerical observations in Fig. 5 .

The Hamiltonian in the "l-bits" basis reads

$$
H=\sum_{i} h_{i} \tau_{i}^{z}+\sum_{i j} J_{i j} \tau_{i}^{z} \tau_{j}^{z}+\cdots
$$

where $J_{i j}$ decays exponentially with the distance between $i$ and $j$. For simplicity and w.l.o.g., we will ignore threeor more-body terms in the Hamiltonian.

We are going to compute the expectation value of $\left[\tau_{1}^{x}(t), \tau_{j_{0}}^{x}\right]$ on product states of "l-bits" in the $Y$ direction. Note that the expectation value on product states in the $Z$ direction is clearly zero, reflecting the absence of transport in eigenstates of the Hamiltonian. By using product states in the $Y$ direction, we hope to access the off-diagonal elements of the commutator although our result shows that the linear response of such states still falls short of achieving the unbounded light cone.

The time evolution of $\tau_{1}^{x}$ is given by

$$
\begin{aligned}
& e^{i H t} \tau_{1}^{x} e^{-i H t} \\
& =e^{i t h_{1} \tau_{1}^{z}} e^{i t \sum_{j} J_{1 j} \tau_{1}^{z} \tau_{j}^{z}} \tau_{1}^{x} e^{-i t \sum_{j} J_{1 j} \tau_{1}^{z} \tau_{j}^{z}} e^{-i t h_{1} \tau_{1}^{z}} \\
& =\left(\cos \left(t h_{1}\right)+i \sin \left(t h_{1}\right) \tau_{1}^{z}\right) \\
& \times \prod_{j}\left(\cos \left(t J_{1 j}\right)+i \sin \left(t J_{1 j}\right) \tau_{1}^{z} \tau_{j}^{z}\right) \\
& \times \tau_{1}^{x} \prod_{j}\left(\cos \left(t J_{1 j}\right)-i \sin \left(t J_{1 j}\right) \tau_{1}^{z} \tau_{j}^{z}\right) \\
& \times\left(\cos \left(t h_{1}\right)-i \sin \left(t h_{1}\right) \tau_{1}^{z}\right) .
\end{aligned}
$$


Conjugating $\tau_{1}^{x}$ with $\prod_{j}\left(\cos \left(t J_{1 j}\right)+i \sin \left(t J_{1 j}\right) \tau_{1}^{z} \tau_{j}^{z}\right)$ generates the following terms:

$$
\tau_{1}^{x}, \tau_{1}^{y} \tau_{j}^{z}, \tau_{1}^{x} \tau_{j}^{z} \tau_{k}^{z}, \tau_{1}^{y} \tau_{j}^{z} \tau_{k}^{z} \tau_{l}^{z}, \ldots
$$

Conjugating these terms with $\left(\cos \left(t h_{1}\right)+i \sin \left(t h_{1}\right) \tau_{1}^{z}\right)$ generates

$$
\tau_{1}^{x \prime}, \tau_{1}^{y^{\prime}} \tau_{j}^{z}, \tau_{1}^{x \prime} \tau_{j}^{z} \tau_{k}^{z}, \tau_{1}^{y^{\prime}} \tau_{j}^{z} \tau_{k}^{z} \tau_{l}^{z}, \ldots,
$$

where $\tau_{1}^{x \prime}$ is the result of rotating $\tau_{1}^{x}$ by $\left(\cos \left(t h_{1}\right)+\right.$ $\left.i \sin \left(t h_{1}\right) \tau_{1}^{z}\right)$ and $\tau_{1}^{y^{\prime}}$ is the result of rotating $\tau_{1}^{y}$ by $\left(\cos \left(t h_{1}\right)+i \sin \left(t h_{1}\right) \tau_{1}^{z}\right)$.

The commutator of these terms with $\tau_{j_{0}}^{x}$ involves terms

$$
\tau_{1}^{y^{\prime}} \tau_{j_{0}}^{y}, \tau_{1}^{x \prime} \tau_{j_{0}}^{y} \tau_{k}^{z}, \tau_{1}^{y^{\prime}} \tau_{j_{0}}^{y} \tau_{k}^{z} \tau_{l}^{z}, \ldots
$$

The expectation value of these terms on product states in the $Y$ direction is nonzero only for the first term. The the bare expectation value (up to \pm 1 ) is $\cos \left(2 t h_{1}\right)$. The norm of this term is $\tan \left(2 t J_{1 j_{0}}\right) \prod_{k} \cos \left(2 t J_{1 k}\right)$. Putting together, the expectation value of the commutator on product state of the "l-bits" in the $Y$ direction is $\cos \left(2 t h_{1}\right) \tan \left(2 t J_{1 j_{0}}\right) \prod_{k} \cos \left(2 t J_{1 k}\right)$.

When $t J_{1 j_{0}} \ll 1$, the second factor is proportional to $t J_{1 j_{0}}$ and hence the expectation value decays exponentially with increasing $j_{0}$. One might want to think of this as the outside of the light cone. However, the expectation value on the boundary of the supposed light cone $j_{c} \sim \log t$ also decays with $j_{c}$, because $\cos \left(2 t J_{1 k}\right)<1$ when $k<j_{c}$ and $\cos \left(2 t J_{1 k}\right) \sim 1$ when $k>j_{c}$. Therefore, the supposed light cone "faints out" with distance. This is exactly the behavior we see in Fig. 5 (lower panels) that there seems to be a logarithmically growing light cone but it disappears with time. Because of this exponential decay of expectation values at light cone boundaries, the expectation value for any $j$ and $t$ can be bounded by an exponentially decaying function of $j$, indicating that there is in fact a localized light cone. If we calculate the maximum achievable expectation value for any $j$, it decays exponentially, similar to that shown in the upper right panel of Fig. 5 . 